BIOCYT Biología, Ciencia y Tecnología, 10(37): 672-686, 2017 (enero-marzo)

ISSN 2007-2082

www.iztacala.unam.mx/biocyt

Publicada en la Facultad de Estudios Superiores Iztacala, Universidad Nacional Autónoma de México

\title{
ASPECTOS DEL COMPORTAMIENTO DE GRAPSUS GRAPSUS (LINNAEUS, 1758) EN LA ZONA INTERMAREAL DE PUERTO VALLARTA, JALISCO, MÉXICO
}

\section{BEHAVIOUR OF GRAPSUS GRAPSUS (LINNAEUS; 1758) IN THE INTERMAREAL ZONE ON PUERTO VALLARTA, JALISCO, MEXICO}

\author{
Sandra Quetzalli Bárcenas-Gutiérrez ${ }^{*}$ y Erick Emmanuel Jáuregui-Velázquez \\ Módulo de Manejo de Recursos Naturales, Facultad de Estudios Superiores Iztacala, Universidad Nacional \\ Autónoma de México. Av. De los Barrios, No. 1, Los Reyes Iztacala, Tlalnepantla, Estado de México, México. \\ C.P. 54090. * quetzy91@hotmail.com, javenoctiluca@hotmail.com
}

\begin{abstract}
The social and reproductive behaviour of G. grapsus are related to aggressive demonstrations. These interactions are common in the acquisition or defence of resources. In this study was observed the behaviour of a crabs population and its relation to the frequency of mutilation in Puerto Vallarta, Jalisco. $93.16 \%$ of the total recording time, the organisms remained standing $(20.14 \%)$, feeding $(19.27 \%)$, walking $(16.98 \%)$ or in the refuge $(32.08 \%)$. Only $5.21 \%$ of the time corresponds to aggressive behaviours, since the first reaction to an aggression is run away, only $1.88 \%$ of the recording time the organisms were fighting. The sex ratio was $1: 0.64$ males/females. $62.50 \%$ of the females and $40 \%$ of the males showed mutilations, but there was no significant difference between females and males. However, in the months of August and July the males presented greater mutilation that was related to the reproductive period. During the reproduction and in general for the activities of G. grapsus, the displacement is characterized by the jumps that it makes, reason why the number of mutilations directly affects in the survival of the organism.

Key words: antagonist, behaviour, conduct, filming, Grapsus, mutilation, jumps, carving.
\end{abstract}

Manuscrito recibido el 12 de septiembre de 2016 aceptado el 06 de diciembre de 2016. 


\section{RESUMEN}

El comportamiento social y reproductivo de G. grapsus está relacionado con conductas agresivas. Estas interacciones son comunes en la adquisición o defensa de recursos. En este trabajo se observó el comportamiento de una población de cangrejos y su relación con la frecuencia de mutilación en Puerto Vallarta, Jalisco. En el $93.16 \%$ de la filmación total, los organismos permanecían de pie (20.14\%), alimentándose (19.27\%), caminando (16.98\%) o en el refugio (32.08\%). Solo el $5.21 \%$ del tiempo de filmación corresponde a conductas agresivas, ya que la primera reacción ante una agresión es la huida; solo el $1.88 \%$ del tiempo fueron enfrentamientos. La proporción sexual fue 1:0.64 machos/hembras. El $62.50 \%$ de las hembras y el $40 \%$ de los machos presentaron mutilaciones, pero no hubo diferencias significativas entre el número de mutilaciones entre hembras y machos; sin embargo en los meses de agosto y julio los machos presentaron mayor mutilación que se relacionó con la época reproductiva. Durante la reproducción y en general para las actividades de G. grapsus, el desplazamiento se caracteriza por los saltos que realiza, por lo que el número de mutilaciones afecta directamente en la supervivencia del organismo.

Palabras clave: antagonista, comportamiento, conducta, filmación, Grapsus, mutilación, saltos, talla.

\section{INTRODUCCIÓN}

Las poblaciones responden a variaciones que son resultado de las "historias de vida" de los organismos dándoles características morfológicas, fisiológicas y etológicas (Moreno, 2001). La importancia de los estudios etológicos radica en que el comportamiento juega un papel importante en la abundancia y la distribución de los organismos, por lo que tiene gran impacto en las prácticas de conservación en estudios de preservación (Cassini, 1999; de Sá Leitão y de Araújo, 2014).

Los animales como las personas son seres sociales, por lo que los estudios de comportamiento han sido usados principalmente para la domesticación de organismos (Cassini, 1999; Petryna y Bavera, 2002).

En general los crustáceos son enfoque de estudios de comportamiento (ICMyL, 2003). Dentro del orden Decápoda se encuentra la familia Grapsidae compuesta por 93 especies aceptadas que ocupan una gran variedad de hábitats (Hernández et al., 2010; WORMS, 2017) que van desde los ambientes intermareales hasta los bosques tropicales. Esta capacidad está asociada al complejo comportamiento social que presentan, caracterizado por una gran variedad de señales y demostraciones ritualizadas (Seiple y Salmon, 1982; Romero, 2003; Gianuca y Vooren, 2007).

Dentro de este género se encuentra Grapsus grapsus (Linnaeus, 1758), es una especie poco estudiada a pesar de su amplia distribución (Romero, 2003; Fernández, 2012), se localiza en latitudes tropicales en el Atlántico y Pacífico Americano (de Florida hasta Brasil y de California hasta Chile) incluyendo las islas Galápagos; Madagascar y el oeste de África. En México se ha reportado su presencia tanto en el golfo de México, como en el Pacifico (Romero y Mutsunori, 1994; Zúñiga, 2002; Romero, 2003; Gianuca y Vooren, 2007; Fernández, 2012; WORMS, 2017). Estos organismos son abundantes en hábitats marinos y juegan un importante papel ecológico, debido al lugar que ocupan en la cadena trófica (Ramírez, 1988; Mantel, 1990; Vinuesa, 2005); en la costa de Jalisco, México, forman parte de la dieta de Crocodylus acutus Cuvier, 1807 (Casas-Andreu y Barrios, 2003).

El cangrejo de las rocas (G. grapsus) (ITIS, 2017; WORMS, 2017) es un gran depredador de las orillas rocosas, presenta hábitos carnívoros y se alimenta en periodos de marea baja. Su dieta está basada en cirrípedos, mitílidos, otros invertebrados y algas. filamentosas (Ramírez, 1988). Son carroñeros, se consideran organismos generalistas y la depredación que realizan estos organismos 
es importante, ya que mantienen áreas desnudas que pueden ser colonizadas por organismos sésiles (Gianuca y Vooren, 2007; Fernández, 2012).

Las poblaciones responden a variaciones que son resultado de la evolución, adaptación y selección de los individuos. G. grapsus presenta autotomía (capacidad voluntaria de perder un apéndice) y canibalismo, estas características en los artrópodos tiene importancia en el balance energético de la especie, ya que sirve de estrategia para mejorar su adecuación y eficacia biológica (Romero, 2003; Mejía-Ortiz, 2010). Por otra parte, las interacciones agonísticas se deben a la adquisición o defensa de recursos como: alimento, refugio o pareja. G. grapsus (y en general los organismos de la familia Grapsidae) se caracterizan por presentar un comportamiento agresivo y territorial. El comportamiento agonístico en la especie comprende despliegues sin un contacto físico, choque de quelas y luchas intensas, que pueden ocasionar la muerte de los individuos (Hernández-Benítez, 2007; Fernández, 2012).

Fernández (2012) observó que el tamaño de un individuo determina el tipo de reacción que tiene en presencia de otro, los organismos de mayor tamaño son los que generalmente muestran conductas agresivas.

Una baja variedad de alimentos, ausencia de depredadores de grandes cangrejos y el aislamiento geográfico, son los factores que determinan las características biológicas y conductuales observadas en G. grapsus. En etapa juvenil los machos presentan tallas mayores de longitud de caparazón $(51.4 \mathrm{~mm})$ que las hembras $(33.8 \mathrm{~mm})$, pero al llegar a la madurez fisiológica el tamaño es similar (machos $38.4 \mathrm{~mm}$ y hembras $33.4 \mathrm{~mm}$ ). G. grapsus presenta diferencias de tamaño entre machos y hembras, siendo éstas las de mayor talla; el dimorfismo sexual permite conocer la talla de madurez, la que se alcanza alrededor de los $35 \mathrm{~mm}$. Los machos poseen un abdomen estrecho a diferencia de las hembras que presentan un abdomen ancho, ya que es usado para almacenar los huevecillos, en los machos las quelas son más anchas que en las hembras (Romero y Mutsunori, 1994).

Los patrones de color están relacionados con la madurez sexual (Freire et al., 2011). Esta especie se caracteriza por su agilidad al moverse o al saltar de roca a roca, también pueden subir por gradientes verticales (Mantel, 1990; de Sá Leitão y de Araújo, 2014).

Los estudios de comportamiento en $G$ grapsus están enfocados en las reacciones agresivas; sin embargo, se sabe que los organismos evitan el enfrentamiento, debido a que representa un desgaste energético, por lo que el objetivo de este trabajo fue describir el comportamiento de una población de G. grapsus en la línea costera de Puerto Vallarta, Jalisco.

\section{MATERIALES Y MÉTODOS}

Área de estudio

El estudio se realizó en la costa de Puerto Vallarta, Jalisco, México, ubicado en bahía de Banderas. Comprende la parte suroeste de la costa de Nayarit y la parte noroeste de Jalisco, tiene un clima cálido subhúmedo con lluvias en verano (A w1 (w)), la temperatura anual promedio es de 26 a $28{ }^{\circ} \mathrm{C}$ y el promedio anual de lluvias es de 930.8 a $1668.0 \mathrm{~mm}$ (Gómez-Graciano y CupulMagaña, 2001).

Se realizaron cuatro muestreos entre agosto de 2013 y julio de 2014 (agosto, octubre de 2013 y abril y julio de 2014) con duración de cuatro días cada uno, en un horario entre 11:00 y 18:00 horas, el principal motivo para delimitar las observaciones, fue la intensidad del oleaje (Romero, 
2003), durante la bajamar los organismos se encuentran en la superficie de la zona rocosa y se observa la mayor actividad (Gianuca y Vooren, 2007; Fernández, 2012). Se establecieron tres puntos de observación: playa Camarones (P1) $\left(20^{\circ} 37^{\prime} 16.1^{\prime \prime} \mathrm{N}, 105^{\circ} 13^{\prime} 56.5^{\prime \prime} \mathrm{W}\right)$, malecón de Puerto Vallarta (P2) $\left(20^{\circ} 36^{\prime} 54.2^{\prime \prime} \mathrm{N}, 105^{\circ} 13^{\prime} 59.5^{\prime \prime} \mathrm{W}\right)$ y playa Las Glorias (P3) $\left(20^{\circ} 37^{\prime} 33.1^{\prime \prime} \mathrm{N}, 1^{\circ} 15^{\circ} 13^{\prime} 56.6^{\prime \prime} \mathrm{W}\right)$. Se realizaron observaciones en acúmulos de rocas ya que los organismos se encuentran en superficies rocosas y cantos próximos a la rompiente de la zona supralitoral (Fig. 1).

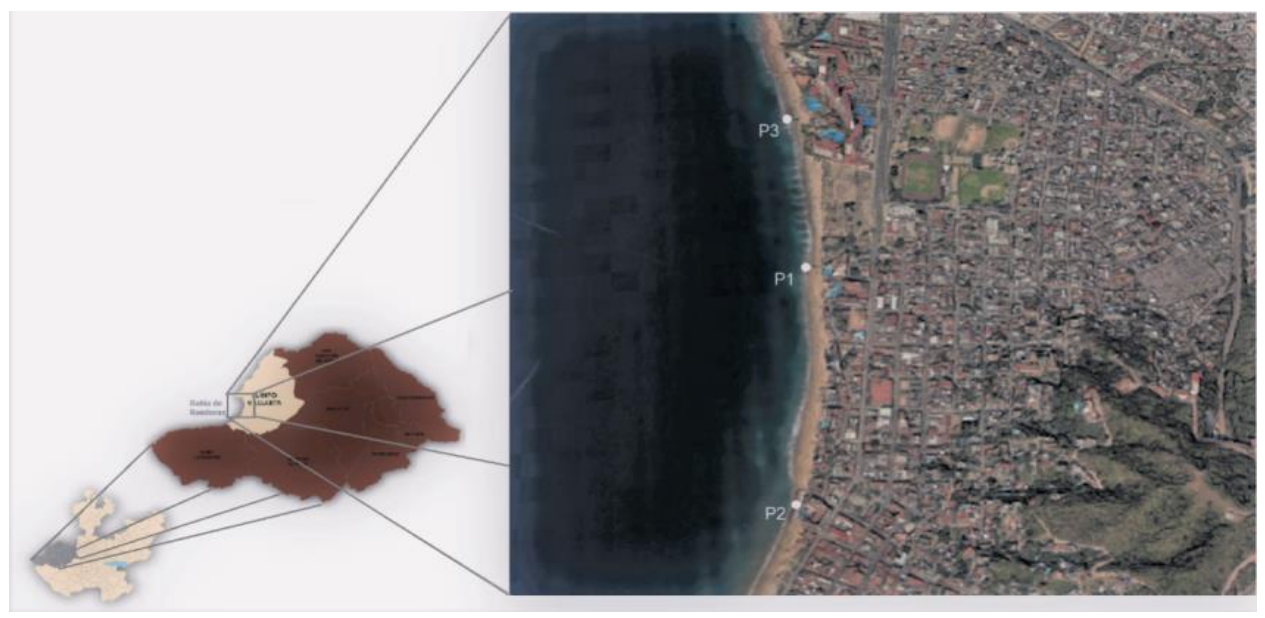

Fig. 1. Puntos de observación: P1 y P2 corresponden a playa Las Glorias y playa Camarones, P3 corresponde al malecón de Puerto Vallarta.

Se realizaron filmaciones de organismos (machos y hembras) de la especie G. grapsus (cámara digital Canon-G10) con la finalidad de observar sus conducta (de Sá Leitão y de Araújo, 2014). Se utilizaron dos métodos de muestreo: 1) de barrido; en el que se exploraron grupos completos de individuos con el fin de obtener información del comportamiento, registrando el número de veces que se observaba una conducta determinada, 2) muestreo focal, en el que se observa a un individuo durante un periodo de tiempo y se registra cuánto tiempo invierte un organismo realizando una actividad determinada (Giri y Collins, 2004; Carazo, 2012; De La CruzManjarrez y Vázquez-López 2015). Se siguieron los criterios de Backwell et al. (2006) y Gianuca y Vooren (2007) para establecer las categorías conductuales. Se realizaron capturas de organismos en los tres puntos mediante un método directo (captura manual y con red) y uno indirecto (colecta de mudas). Las colectas se usaron para determinar la proporción sexual y calcular los porcentajes de mutilación presentes en los organismos. Se determinó el sexo y se midieron en ancho y largo de caparazón de los organismos y las mudas colectadas. Debido a que los organismos presentan hasta 60 tallas diferentes (Fernández, 2012) se establecieron seis intervalos de tallas con intervalos de 10 $\mathrm{mm}$ de diferencia, tomando en cuenta el organismo colectado más pequeño y el más grande. Se realizó un registro de los organismos que presentaron mutilaciones (Romero, 2003; Fernández, 2012). Se realizó una prueba U Mann Whitney para observar posibles diferencias entre la frecuencia de mutilaciones entre sexos. Se registró la temperatura y salinidad con un multiparamétrico Oakton 120 (de 0 a 50 UPS). 


\section{RESULTADOS}

Conductas sociales

Muestreo de barrido

Se obtuvieron 36 videos, con duración total de tres horas en los que se filmaron 170 organismos. Se identificaron diez tipos de conductas: (a) de pie (estáticos), (b) alimentándose, (c) caminando, (d) en su refugio, (e) defendiendo el refugio, (f) ahuyentando (a otros cangrejos), (g) agresión y huida, (h) agresión y enfrentamiento, (i) cortejo y copula y (j) desplazamiento mediante saltos (Fig. 2).

En P1 se observó el mayor número conductas registradas (51.57 \%), seguido de P2 con 29.51 \% y finalmente P3 con 18.91 \%. Se registró una conducta agresiva en todos los puntos, también se pudo observar que los agresores generalmente son más grandes que los cangrejos agredidos.

Se identificaron cuatro tipos de conducta antagonista: (f) ahuyentando (ahuyentando a otro cangrejo del refugio o del alimento), (e) defensa del refugio (en ocasiones el dueño del refugio salía repentinamente cuando otro organismo se acercaba a este), (g) agresión y huida y (h) agresión y enfrentamiento (Fig. 3). Este comportamiento se observó con más número de veces en el muestreo 4 (julio) con $73.68 \%$ del total de las conductas agresivas registradas en el estudio.

Los cangrejos G. Grapsus pasan la mayor parte del tiempo (a) de pie sobre las rocas y (b) alimentándose o haciendo ambas actividades simultáneamente (Fig. 3). Los organismos se trasladan de una roca a otra realizando saltos de diferente distancia, desde unos cuantos centímetros hasta saltos de aproximadamente un metro (j). La forma de desplazamiento mediante saltos es común en las actividades que realizan estos cangrejos. Se filmaron 29 saltos a lo largo del estudio. La mayoría de los saltos se observaron en P1 (41.37\%), P2 (34.48\%) y finalmente en P3 (24.13\%). En el muestreo 4 se registró el $44.82 \%$ de los saltos, en el muestreo 1, 31.03\%, en el muestreo 2, 17.24 y en el muestreo 3, 6.88\%.

\section{Muestreo focal}

Se filmó el comportamiento de 48 organismos durante un minuto. A partir del tiempo total (48 min.) se calcularon porcentajes del tiempo que invirtieron los organismos en cada conducta; con base en esto se observó que los cangrejos pasaron 32.08\% del tiempo en su refugio (924 seg), 20.14\% de pie sobre las rocas (580 seg), 19.27\% alimentándose (555 seg), 16.98\% caminando sobre las rocas (489 seg). En este método se consideró otra conducta: en la que el organismo se alimenta mientras camina; en ésta los organismos invirtieron 4.69\% del tiempo (135 seg). Se observó que cuando los organismos están de pie (estáticos) sobre las rocas, pueden permanecer junto a otros organismos de tallas diferentes sin presentar confrontación alguna. Durante el muestreo focal ninguno de los indviduos presentó el comportamiento de defensa del refugio. Se observó que 1.11\% del tiempo los cangrejos lo invierten en ahuyentar a otros individuos con la finalidad de defender su refugio o su alimento, el $2.22 \%$ se presentó la conducta de agresión y huida y solo el $1.88 \%$ del tiempo se dedicó a un enfrentamiento, sin embargo, la mayor parte del tiempo (94.79\%) evitan confrontaciones con otros organismos (Fig. 4). A lo largo de las filmaciones se observaron 11 saltos, los organismos lo usan para desplazarse, huir y durante el cortejo para la cópula. 


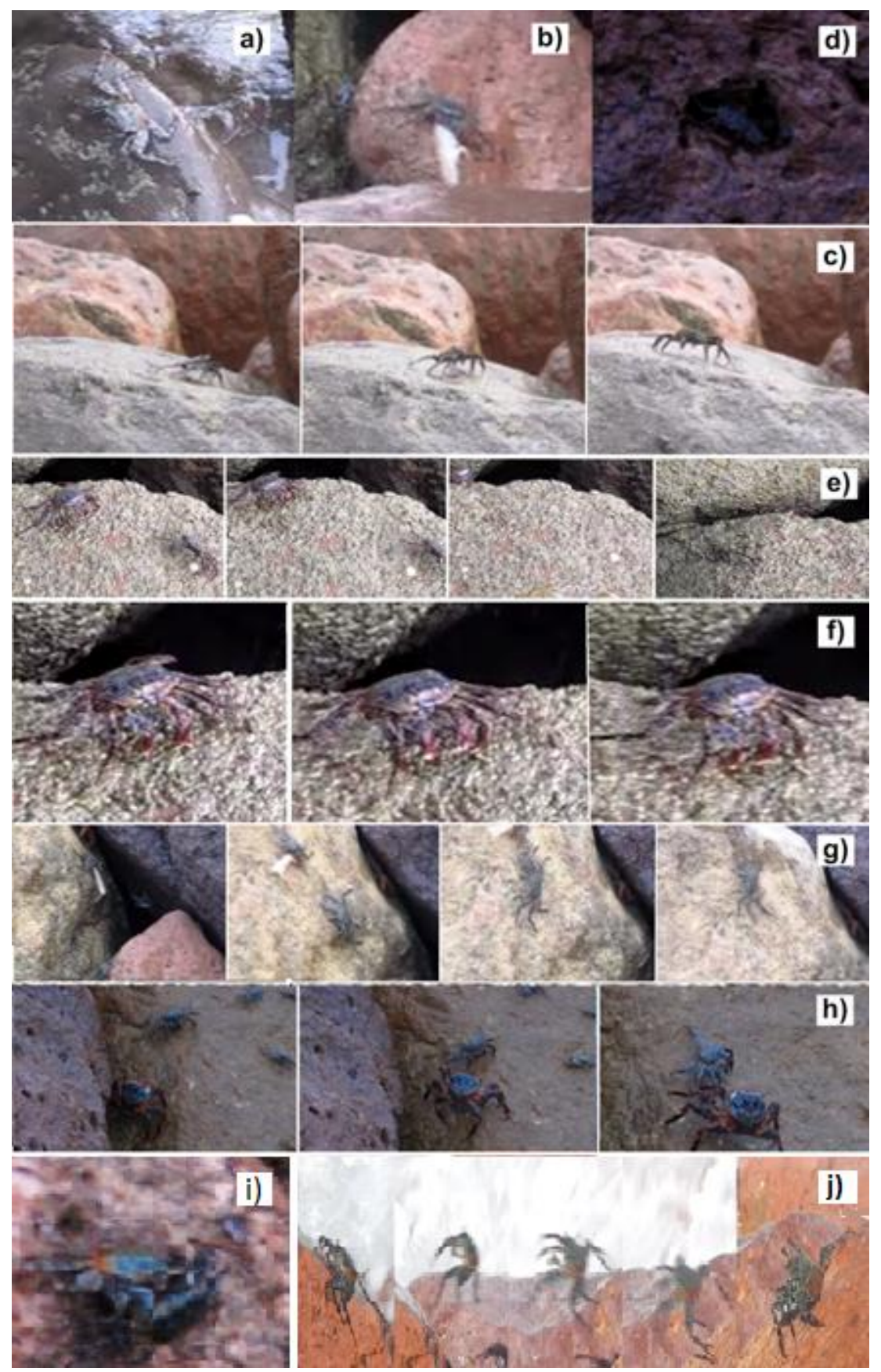

Fig. 2. Tipos de conductas que presenta G. grapsus. (a) de pie; (b) alimentándose; (c) caminando, (d) en su refugio; (e) defendiendo el refugio, (f) ahuyentando, (g) agresión y huida; (h) agresión y enfrentamiento; (i) copula y (j) desplazamiento mediante saltos. 


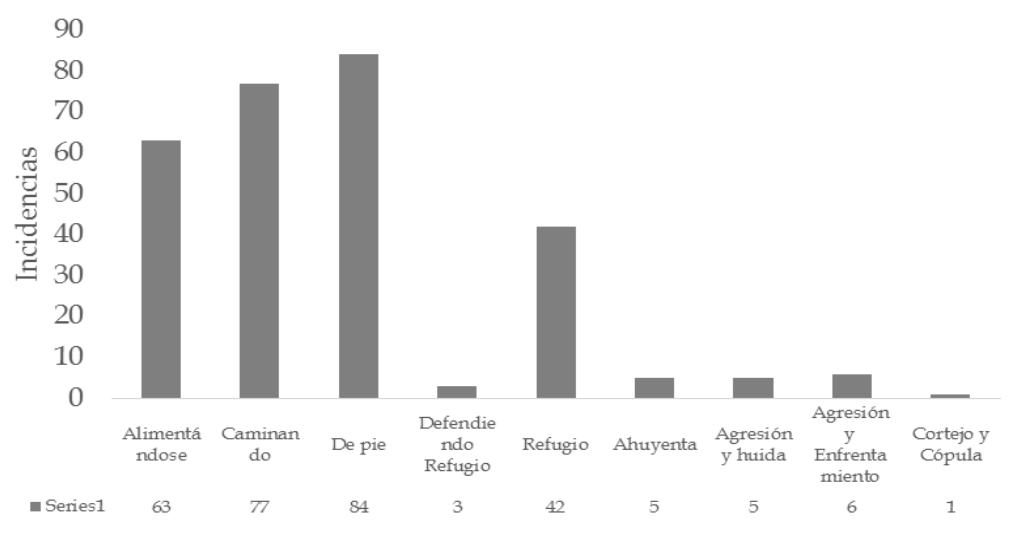

Fig. 3. Frecuencia de observaciones de las conductas identificadas en el muestreo de barrido.

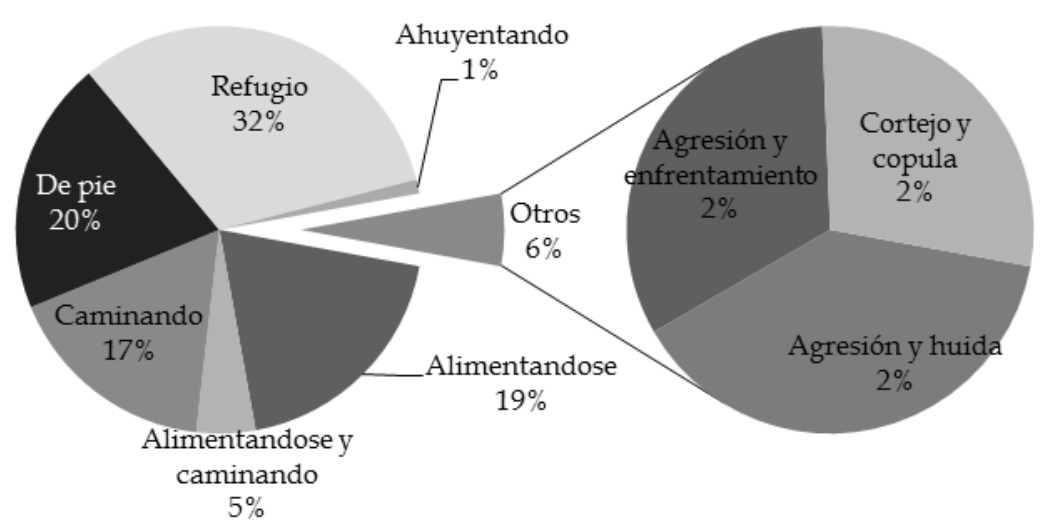

Fig. 4. Tiempo dedicado a cada actividad (en porcentaje) (muestreo focal).

En cada muestreo se filmó un total de 720 segundos ( $240 \mathrm{seg} /$ por punto). En el muestreo 1 se observaron dos saltos; los organismos pasaron $37.64 \%$ del tiempo en su refugio ( $271 \mathrm{seg}$ ), $21.25 \%$ de pie (153 seg), caminando el 18.19\% (131 seg) y alimentándose un 13.33\% (96 seg). Del 4\% del tiempo de grabación en donde se presentaron conductas agresivas se observó que únicamente el $1.39 \%$ del tiempo, los organismos lo dedicaron a un enfrentamiento (10 seg). Se observó una persecución que se caracterizó por ser descontrolada por alrededor de ocho segundos que terminó en cópula durante el muestreo 2. En este muestreo se observaron seis saltos y organismos de tan solo unos milímetros que también presentan pequeños saltos, se cree que el mes de octubre es la época de mayor reclutamiento de la especie; sin embargo se filmó una cópula en octubre.

La cópula observada comenzó una vez que el macho acorraló a la hembra, éste se colocó bajo la hembra a manera de columpio y poniendo sus quelas a la altura de los ojos de la hembra. La duración fue de 2.18 minutos desde el acoplamiento hasta que los individuos se separaron; solo se tomó en cuenta un minuto de la grabación, que para el muestreo 2 representó el $6.53 \%$ del tiempo total (47 seg), sin embargo para la descripción de la conducta se empleó la filmación completa. Posterior al acoplamiento, los organismos se separan y se alejan. Durante ésta cópula no se observó 
ninguna conducta agresiva, ni mutilación. Los organismos pasaron el 15.56\% del tiempo en su refugio (112 seg), 30.83\% de pie (222 seg), caminando el 23.75\% (171 seg) y alimentándose un $14.44 \%$ (104 seg). Respecto a conductas después de una agresión, los organismos consumieron $2.78 \%$ del tiempo huyendo $(20 \mathrm{seg}$ ) y el $5.28 \%$ del tiempo de filmación en un enfrentamiento (38 seg) En el muestreo 3 se observó un salto. Los organismos pasaron el 34.86\% del tiempo en su refugio (251 seg), 23.89\% de pie (172 seg), caminando el 16.11\% (116 seg) y alimentándose 20.69\% (149 seg). Respecto a conductas agresivas, el 1.53\% (11 seg) del tiempo de filmación, los organismos ahuyentaron a otros cangrejos de su refugio o de su alimento, en este muestreo no se observó ningún enfrentamiento ni huida.

En el muestreo 4 se observaron dos saltos, que los organismos pasan $40.28 \%$ del tiempo en su refugio (290 seg), el 4.58\% de pie (33 seg), caminando el 9.86\% (71 seg) y alimentándose un $28.61 \%$ (206 seg). En cuanto a demostraciones agresivas, 2.08\% (15 seg) del tiempo de filmación, los organismos ahuyentaron a otros cangrejos de su refugio o de su alimento, después de una agresión los organismos consumieron $2.92 \%$ del tiempo huyendo y $0.83 \%$ en enfrentamientos (Fig. 5).

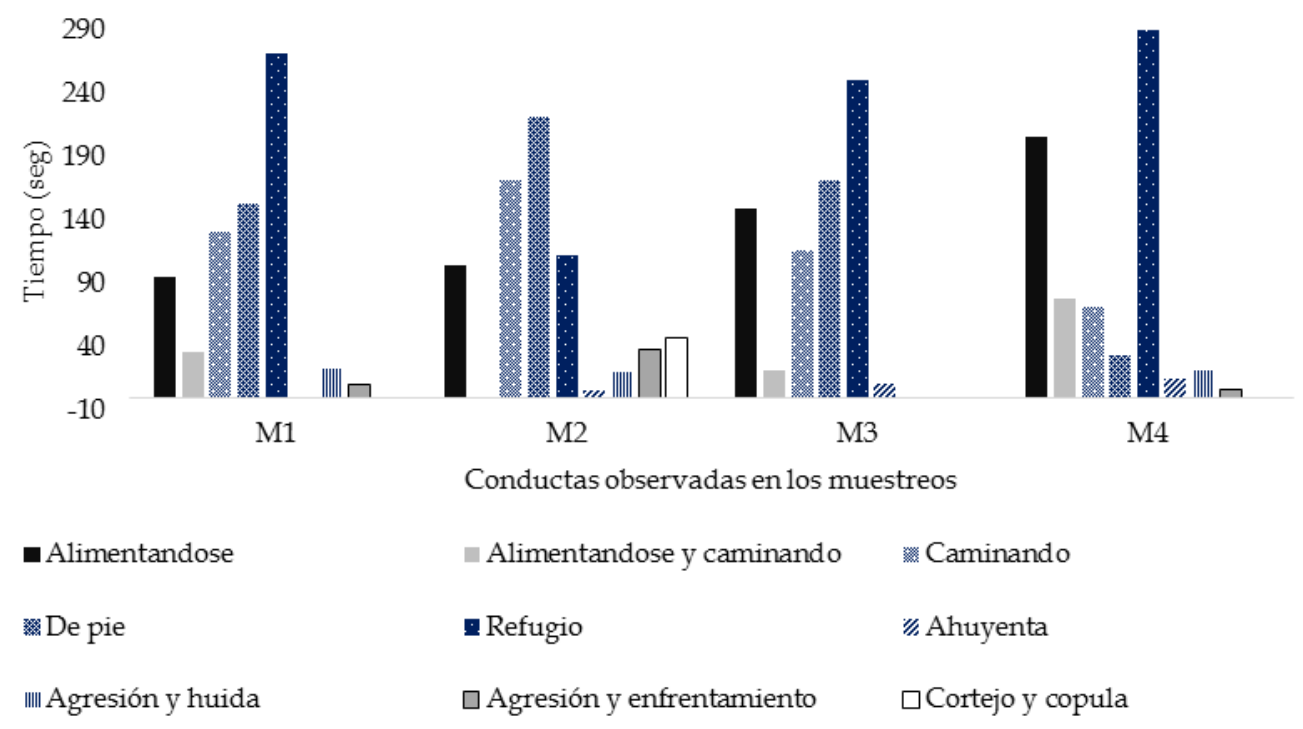

Fig. 5. Distribución del tiempo invertido en las diferentes conductas de G. grapsus en los cuatro muestreos.

Fue posible identificar el sexo de los organismos a partir de $27 \mathrm{~mm}$ de ancho y $25 \mathrm{~mm}$ de largo a pesar de que no están completamente en el intervalo de madurez sexual que se presenta a partir de $30 \mathrm{~mm}$ (Fig. 6).

La relación entre ancho y largo $(\mathrm{mm})$ de los organismos se expresó mediante un modelo de regresión lineal (expresado por la ecuación y $=0.8606 x+1.0435$ ) con $n=23$ y $r=0.986$; el modelo se ajustó en un $97.433 \%$ explicando la relación que existe entre el ancho y el largo del caparazón con $\mathrm{p}<0.05\left(\mathrm{R}^{2}=0.9548\right)$ (Fig. 7). 

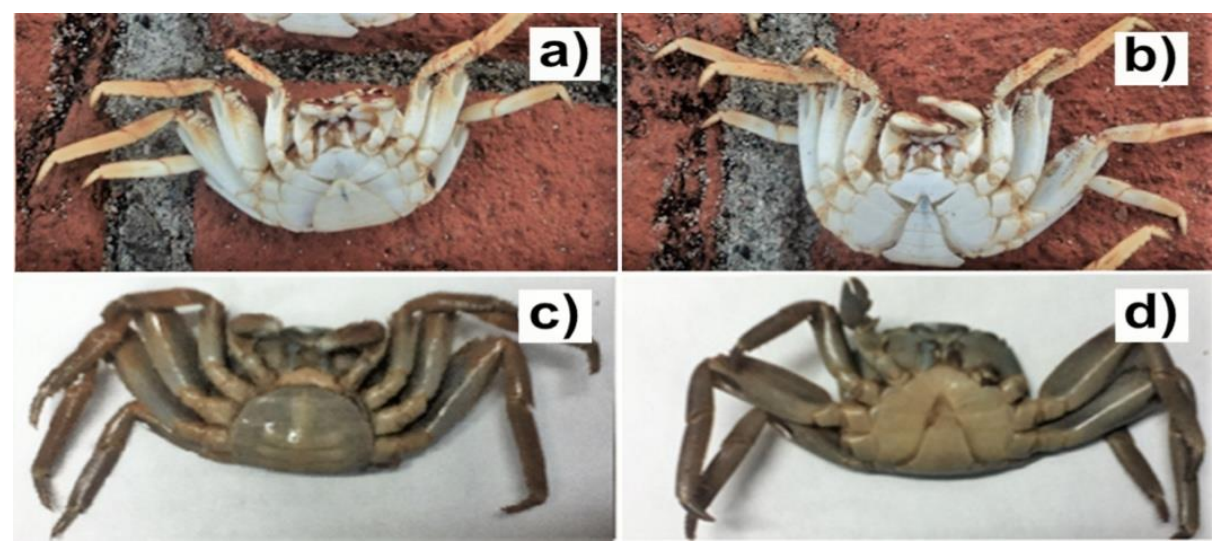

Fig. 6. Dimorfismo sexual. a) Muda de hembra de $28 \mathrm{~mm}$ de ancho, b) muda de macho de $29 \mathrm{~mm}$ de ancho, c) hembra de $51 \mathrm{~mm}$ ya diferenciada y d) macho de $60 \mathrm{~mm}$ ya diferenciado.

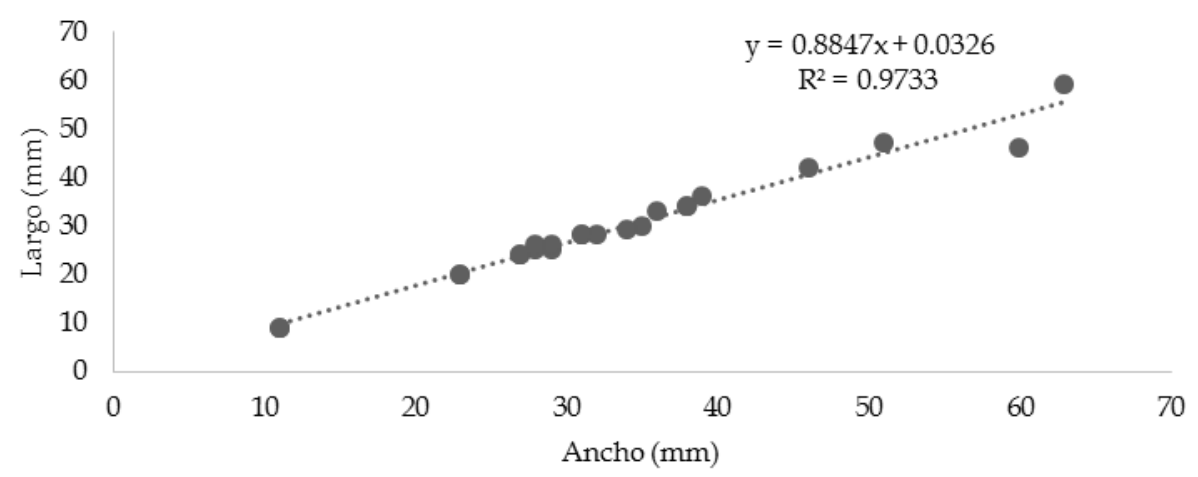

Fig. 7 Relación entre ancho y largo (mm) de caparazón.

Se recolectaron 12 organismos y 11 mudas (seis organismos y ocho mudas pertenecientes a machos y seis organismos y tres mudas de hembras). En los muestreos 1 (agosto), 2 (octubre) y 4 (julio) se colectaron cuatro, seis y tres, en el muestreo 3 (abril) se colectaron dos hembras y un macho (Fig. 8). La proporción sexual fue 1:1 machos/hembras y en mudas 1:0.37 machos/hembras. En el muestreo 2 (octubre) se encontraron más mudas. La proporción sexual total (organismos y mudas) fue 1:0.64 machos/hembras.

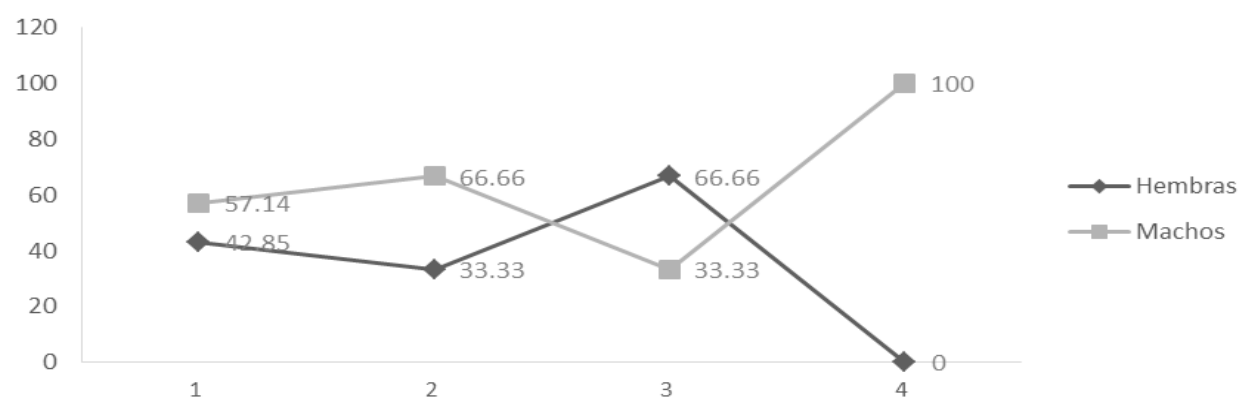

Fig. 8. Porcentaje de hembras y machos en cada muestreo. 
La prueba U de Mann Whitney no mostró diferencias significativas entre las tallas de ambos $\operatorname{sexos}(\mathrm{Z}=-0.58$ y $\mathrm{p}=0.56$ ancho $)(\mathrm{Z}=-0.61 \mathrm{y} \mathrm{p}=0.53$ largo $)$.

El 52.17\% de los organismos presentaron algún tipo de mutilación (siete machos y cinco hembras). Se agruparon por muestreo y se contrastaron las proporciones de machos y hembras. El $62.50 \%$ de las hembras y $40 \%$ de los machos colectados presentaron algún tipo de mutilación. Las hembras presentaron mayor número de mutilaciones que los machos en el muestreo 3 (abril), en el muestreo 2 (octubre) se observó el mismo número de machos y hembras con mutilaciones y en agosto y julio los machos presentaron mayor número de mutilaciones que las hembras (Fig. 10).

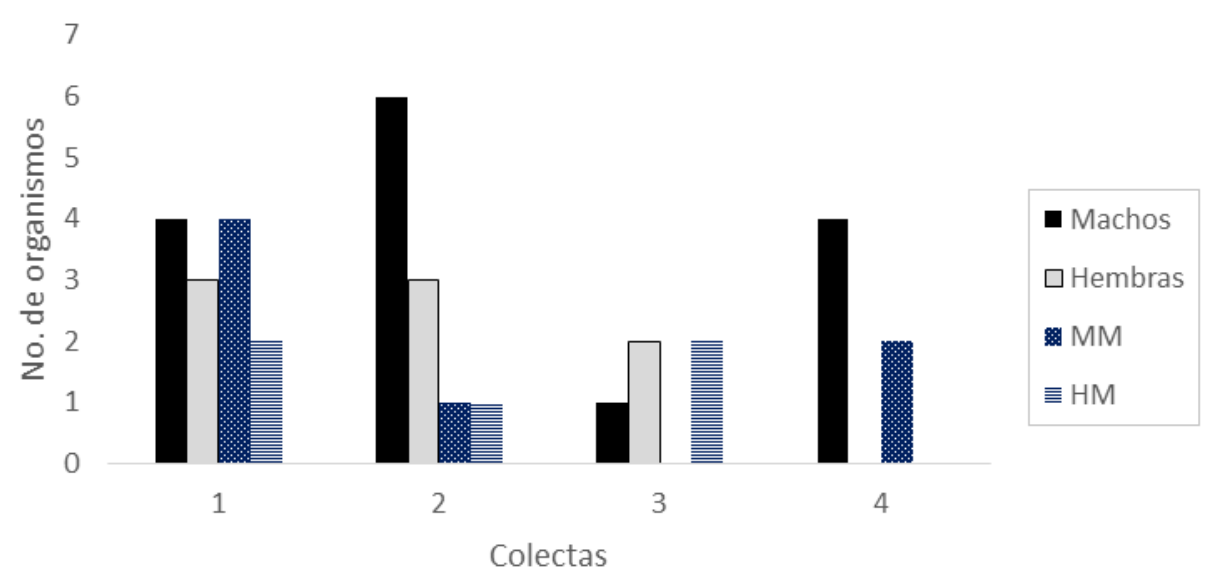

Fig. 10. Número de machos y hembras colectados y número de machos (MM) y hembras con mutilaciones (HM).

El organismo colectado de menor talla fue de $11 \mathrm{~mm}$ de ancho y $9 \mathrm{~mm}$ de largo de caparazón (indiferenciado sexualmente) y el de mayor talla fue un macho de $63 \mathrm{~mm}$ de ancho y 59 de largo. Las dos primeras tallas estuvieron representadas por cuatro organismos indiferenciados por lo tanto no se tomaron en cuenta para estimar la proporción sexual. A partir de la talla 3 a la 6 , las hembras presentaron mayor número de mutilaciones (de 1 a 5 apéndices mutilados), el porcentaje promedio es $18.75 \%$ de apéndices mutilados, en machos se observó $9.33 \%$ (1 a 3 apéndices mutilados) de apéndices mutilados (Fig. 11 a y Fig. 11 b).

a)

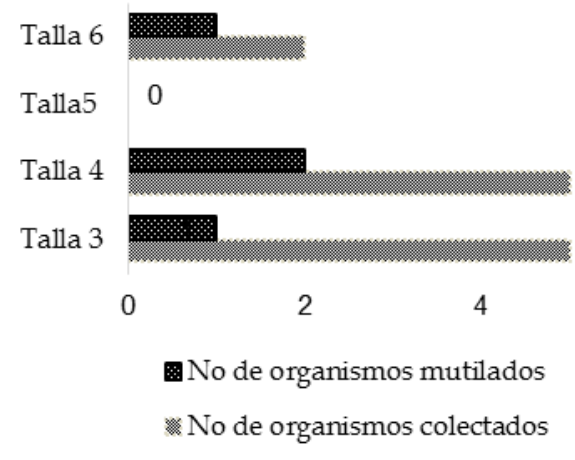

b)

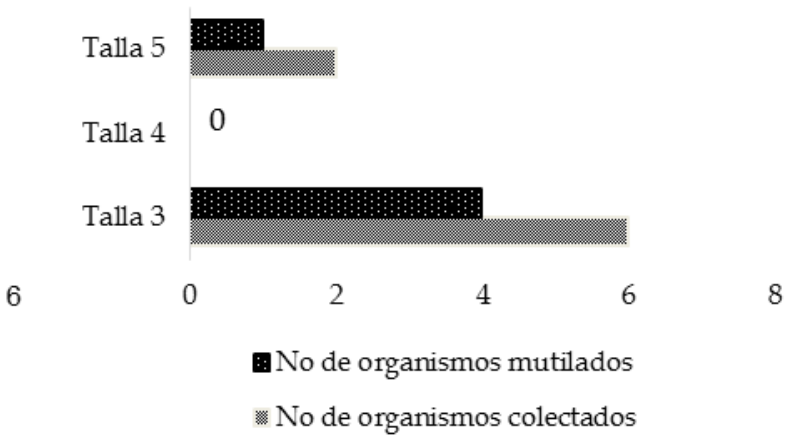

Fig. 11. Distribución de tallas de G. grapsus. a) machos y b) hembras. Las barras gris claro representan el total de organismos colectados. 
La prueba U Mann Whitney no mostro diferencias significativas entre la frecuencia de mutilaciones entre sexos $(Z=0.92$ y $\mathrm{p}=0.35)$.

\section{DISCUSIÓN}

El gobierno de Jalisco (2017) ha reportado que a partir de la década de 1990 la zona urbana de Puerto Vallarta ha ido creciendo. Por lo que es importante promover la conservación de las especies. El impacto de la etologia (como disciplina) en las prácticas de preservación se debe a que de esta forma se conocen las fuentes naturales de variación (Cassini, 1999). En las zonas cercanas al malecón de Puerto Vallarta y al borde de una playa privada (en el mismo puerto) se observó una menor actividad de los organismos a lo largo del estudio, esto quizá relacionado con la presencia humana.

Las conductas antagonistas han sido las más estudiadas en G. grapsus (Romero 2003; Gianuca y Vooren, 2007; Freire et al., 2011; Fernández, 2012); en este estudio se identificaron cuatro tipos de conductas antagonistas: ahuyentando; defensa del refugio; agresión y huida; agresión y enfrentamiento. Las demostraciones agresivas que presenta esta especie consisten en movimientos verticales con las quelas con las puntas hacia abajo, esta conducta también fue descrita por Romero (2003), junto con la demostración agresiva cuando llegan a un enfrentamiento en donde los organismos se mueven de atrás, hacia adelante y entrelazando los apéndices; sin embargo, en este estudio se observó que los individuos pasaron la mayor parte del tiempo de píe (estáticos), caminando, alimentándose o en su refugio. Gianuca y Vooren (2007) mencionan que en los registros conductuales predomina la conducta "alimentándose".

Romero (2003) y Freire et al. (2011) mencionan que la época reproductiva de G. grapsus ocurre en verano y que presenta una conducta agresiva durante la reproducción. Romero (2003) indicó que el $7.4 \%$ de las cópulas terminaron en la muerte de uno de los individuos. En este estudio se observó la mayor actividad y un número considerable de organismos que presentaron mutilaciones en los muestreos que corresponden a la época reproductiva. Durante el cuarto muestreo (julio) se observó la mayor actividad antagonista, esto se puede explicar por la época reproductiva, que como en el caso de Chasmagnathus granulata Dana, 1851 (Neohelice granulata Dana, 1851), inicia en verano (hemisferio sur), sin embargo se puede afirmar que los organismos se reproducen todo el año ya que la cópula que se filmó ocurrió en octubre. Collins et al. (2004), observaron que las persecuciones son comunes en crustáceos no solo en época reproductiva, también se pueden observar en la captura de presas; López y Rodríguez (1998) observaron que en la especie G. grapsus las persecuciones son descontroladas y que terminan en cópula, esto es similar a las persecuciones que se observaron en este estudio. Se observó una persecución que concluyó en cópula en la que ambos participantes realizaron saltos para desplazarse entre las rocas. Romero (2003) presenció cinco persecuciones e identificó la más prolongada por más de $50 \mathrm{~m}$.

Se observó que los organismos pueden permanecer inmóviles o alimentándose sobre las rocas en grupos de hasta 16 individuos, Romero (2003) observó grupos de hasta 60 individuos en el mismo sitio. Fernández (2012) establece que ciertos animales que viven en grupos suelen evitar el riesgo de enfrentamientos porque representan un desgaste energético; sin embargo, por medio de esto se pueden establecer relaciones de dominancia que forman incluso jerarquías sociales; Hernández-Benítez (2007) concluyó que ante una agresión continua, los organismos de menor tamaño desisten y huyen; esto también se observó en el presente trabajo. Generalmente los organismos más pequeños evitan acercarse a los organismos de mayor tamaño; en ocasiones se 
acercan con el fin de enfrentarse, cuando hay alimento de por medio o cuando defienden su refugio.

A pesar de que los organismos presentan una conducta agresiva se observó que la mayoría de las veces evitan llegar al enfrentamiento; siendo esto el último recurso. Freire et al. (2011), indicaron que la talla es determinante en los combates y es más probable que ocurran si los combatientes presentan un tamaño similar.

En miembros de la familia Grapsidae como Pachygrapsus marmoratus Fabricius, 1787 se observa un incremento de la agresividad respecto a la proximidad de intrusos y la defensa del refugio, ésta conducta se observó en el presente trabajo: los organismos ahuyentaban a otros cangrejos si estos se aproximaban demasiado. Romero 2003 indica que a pesar de que estos organismos son altamente territoriales pueden desplazarse sobre pasando los límites de sus refugios.

Se observó que tanto hembras como machos juveniles y adultos presentan la habilidad de saltar, esto fue descrito por de Sá Leitão y de Araújo (2014), estos autores mencionan que los saltos están relacionados con una actitud defensiva. En las filmaciones realizadas se muestra que los organismos son muy ágiles y saltan con frecuencia para evadir el oleaje, perseguirse en época reproductiva, evadir a otros miembros de la población o simplemente para desplazarse entre las rocas e incluso pueden saltar al mar para evitar un enfrentamiento o ante la presencia de un depredador. Chelazzi y Vannini (1987) mencionan que G. grapsus puede saltar gracias a la condición física activa subarterial de locomoción y sensorial.

Proporción de sexos

En el periodo de estudio la proporción sexual favoreció a los machos, a diferencia de lo reportado por Fernández (2012), quien además estableció que no existen diferencias entre la incidencia de mutilaciones entre sexos, aun así, los machos presentaron mayor número de mutilaciones en el mes de julio, esto debido a que en la época reproductiva se incrementan los enfrentamientos, lo que aumenta la posibilidad de sufrir mutilaciones o la pérdida total de algún apéndice.

Tallas

No se observaron diferencias entre las tallas de hembras y machos, pero autores como Romero y Mutsunori (1994) y Fernández (2012) sí observaron diferencias de tamaño entre sexos en la misma especie, siendo las hembras las de mayores tallas. En esta especie el dimorfismo sexual permite conocer que la talla de madurez sexual se presenta alrededor de los $35 \mathrm{~mm}$, en la literatura se ha reportado que en organismos juveniles, los machos presentan tamaños mayores.

Los machos poseen un abdomen estrecho a diferencia de las hembras (cercanas a la etapa reproductiva) que poseen el abdomen ancho, ya que es usado para almacenar los huevecillos; Freire et al. (2011), ubican la madurez fisiológica en un tamaño similar para ambos sexos, en el presente estudio se pudo establecer que las hembras alcanzan esta etapa a partir de los $26 \mathrm{~mm}$.

La mayoría de los organismos observados presentan tonalidades gris-azuladas, mientras que los de mayor tamaño presentan colores brillantes rojos y anaranjados, lo que indica que la madurez sexual también está relacionada con el color (Freire et al., 2011).

Las respuestas agresivas se dan por la disputa de recursos como: alimento, refugio, espacio o bien una pareja reproductiva. Los organismos pueden permanecer de pie en grupos de 
individuos de número variado, lo que indica que podría existir dominancia, incluso se cree exhiben un orden jerárquico, la talla es un factor decisivo en el comportamiento ya que los organismos de mayor talla son los que presentan respuestas más agresivas, los organismos de tallas menores generalmente huyen ante las demostraciones agresivas, con respecto a organismos ajenos al refugio. A pesar de que los organismos están asociados a sus refugios, pueden caminar algunos metros alejándose de él, pero posteriormente regresan. No existieron diferencias significativas en el número de mutilaciones entre sexos. El grado de mutilación que presentan los organismos afecta el comportamiento debido a que la falta de apéndices limita las actividades que realizan los organismos incluyendo su desplazamiento y su capacidad para saltar; lo que los hace más vulnerables a ser depredados. Se puede concluir que el comportamiento de G. grapsus posee una gran variedad de señales y demostraciones ritualizadas. Una conducta agresiva es común en esta especie, sin embargo, los individuos evitan estas interacciones agresivas. Las actividades que realizan varían en tiempo, sin embargo, pasan la mayor parte de pie sobre las rocas, en el refugio o alimentándose.

\section{AGRADECIMIENTOS}

A los miembros pertenecientes al Laboratorio de Ecología y Conservación de la Facultad de Estudios Superiores Iztacala, Universidad Nacional Autónoma de México; principalmente al Dr. Horacio Vázquez López por la supervisión y revisión de este estudio. De igual forma agradecen a los revisores quienes con sus comentarios enriquecieron sustancialmente el manuscrito. Finalmente a las facilidades otorgadas por el personal del estero El Salado.

\section{REFERENCIAS}

1. Backwell P., M. Jennions, K. Wada, M. Murai y J. Christy, 2006. Synchronous waving in two species of fiddler crabs. Acta Ethologica, 9(1): 22-25.

2. Carazo I.O., 2012. Comportamiento reproductivo y fisiología del lenguado senegalés, (Solea senegalensis) en cautividad. (Tesis de Doctorado, Universidad de Barcelona, España).

3. Casas-Andreu G. y G.Q. Barrios, 2003. Hábitos alimenticios de Crocodylus acutus (Reptilia: Crocodylidae) determinados por el análisis de sus excretas en la costa de Jalisco, México. Anales del Instituto de Biología, Serie Zoología, 74(1): 35-42.

4. Cassini M.H., 1999. Importancia de la conservación. Etología, 7: 69-75.

5. Chelazzi G. y M. Vannini, 1987. Behavioral adaptation to intertidal life. Vol. 151, Springer, Florencia, Italia.

6. Collins P.A., V. Williner y F. Giri, 2004. Crustáceos decápodos del litoral fluvial argentino. INSUGEO, Miscelánea, 12: 253-264.

7. De la Cruz-Manjarrez C. y H. Vázquez-López, 2015. Aspectos etológicos del cangrejo violinista Uca latimanus Rathbun, 1893 en el estero el Saldo, Puerto Vallarta, Jalisco, México. Biología, Ciencia y Tecnología 8(31): 557-569. 
8. de Sá Leitão M. y C. de Araújo, 2014. The leaping behavior of the sally lightfoot crab Grapsus grapsus (Crustacea: Decapoda: Brachyura) at an oceanic archipelago. Journal of Research in Biology, 4(4): 1357-1364.

9. Gianuca D. y C.M. Vooren, 2007. Abundance and behavior of the sally lightfoot crab (Grapsus grapsus) in the colony of the brown booby (Sula leucogaster) in the São Pedro and São Paulo Archipelago. Investigaciones Marinas, 35(2): 121-125.

10. Giri F. y P. Collins, 2004. Eficiencia en la captura del camarón dulceacuicola Palaemonetes argentinus (Nobili 1901) sobre larvas de mosquito Culex pipiens s.l. (Linnaeus 1758).Hidrobiología 14(2): 85-90.

11. Gobierno de Jalisco, 2010. http://www.jalisco.gob.mx/wx/jalisco/municipios/puerto-vallarta (accesado en enero 18, 2017).

12. Fernández E.S., 2012. Efecto del color del oponente en el comportamiento agresivo del Grapsus grapsus (Linnaeus, 1758) (Crustacea, Decapoda). Anales Universitarios de Etología, 6: 1-10.

13. Freire A.S., M.A.A.-Pinheiro, H. Karam-Silva y M.M. Teschima, 2011. Biology of Grapsus grapsus (Linnaeus , 1758) (Brachyura, Grapsidae) in the Saint Peter and Saint Paul Archipelago, Equatorial Atlantic Ocean. Helgoland Marine Research, 65(3): 263-273.

14. Gómez-Graciano F.S. y F.G. Cupul-Magaña, 2001. La técnica de análisis de amenazas como herramienta de diagnóstico ambiental: caso Estero El Salado. Ciencia y Mar, 5(14): 33-42.

15. Hernández-Benítez M.A., 2007. Comportamiento agresivo de Pachygrapsus marmoratus (Crustacea, Decapoda) ante competidores simulados. Anales Universitarios de Etología, 1: 32-36.

16. Hernández C., F. Álvarez y JL. Villalobos, 2010. Crustaceans associated to hard substrate in the intertidal zone of Montepío, Veracruz, México. Revista Mexicana de Biodiversidad, 81: 141-151.

17. ICMyL (Instituto de Ciencias del Mar y Limnológia), 2003. Crustáceos. http://www.icmyl.unam.mx/arrecifes/lobsters.html. (accesado diciembre 7, 2016).

18. ITIS (Integrated Taxonomic Information System), 2017.

https:// www.itis.gov/servlet/SingleRpt/SingleRpt\#null (accesado enero 10, 2017).

19. López L.S. y E.M. Rodríguez, 1998. Somatic growth in juveniles of the estuarine crab Chasmagnathus granulate (Brachyura, Grapsidae), under laboratory conditions. Investigaciones Marinas, 26: 127-135.

20. Mantel L.H., 1990. The biology of Crustacea. Internal Anatomy and Physiological Regulation. Vol. 5, Academic Press,Inc., New York.

21. Mejía-Ortiz L.M., 2010. Pautas de comportamiento alimentario y adaptaciones progresivas en los apéndices auxiliares en Agostocaris bozanici y Barbouria yanezi (Crustacea: Decapoda: Caridea: Agostocarididae, Hyppolitidae). Revista Mexicana de Biodiversidad, 81: S193-S201.

22. Moreno C.E., 2001. Métodos para medir la biodiversidad. M yT, Manuales y Tesis SEA, vol. 1., Zaragoza, España. 
23. Petryna A. y G.A. Bavera, 2002. Etología. Cursos de producción bovina de carne, FAV UNRC. http://www.produccion-animal.com.ar/etologia_y_bienestar/etologia_en_general/07etologia.pdf (accesado en agosto 21, 2013).

24. Ramírez M.F., 1988. Contribución al conocimiento de la abundancia de larvas de crustáceos decápodos (Orden: Decápoda) en el Golfo de México. (Tesis profesional, Facultad de Estudios Superiores Iztacala. Universidad Nacional Autónoma de México, México).

25. Romero L., 2003. Reproductive behaviour and mutilations in sally lightfoot Grapsus grapsus (Linnaeus, 1758) (Crustacea, Decapoda). Revista Peruana de Biología, 10(2): 195-202.

26. Romero, L. y T. Mutsunori, 1994. Aspectos del comportamiento del cangrejo Grapsus grapsus (Decapoda, Grapsidae). Resumen presentado a la IV Reunión del Instituto de Investigaciones de Ciencias Biológicas "Antonio Raimondi” (ICBAR).

27. Seiple W. y Salmon M., 1982. Comparative social behavior of two grapsid crabs, Sesarmareti culatum and S. cinereum. Journal of Expimental Marine Biology and Ecology, 64: 1-24.

28. Vinuesa J.H., 2005. Distribution of decapod and stomatopod crustaceans from San Jorge Gulf, Argentina. Revista de Biología Marina y Oceanografía 40(1): 7-21.

29. WORMS, 2013. Grapsus Lamarck, 1801, Aphia ID: 106963. http:// www.marinespecies.org/aphia.php?p=taxdetails\&id=106963\&allchildre

(accesado febrero 02, 2017).

30. Zúñiga O.R., 2002. Guía de Biodiversidad. Vol 1. Macrofauna y algas marinas, No. 2. Crustáceos. Centro regional de Estudios y Educación Ambiental II Región de Antofagasta, Chile. http://svsch.ceachile.cl/e-Biblioteca/Documentos/Biodiversidad/2002_Guia_de_Crustaceos.pdf (accesado diciembre 08, 2016). 